Article

\title{
Possible Occurrence of Superconductivity by the $\pi$-flux Dirac String Formation Due to Spin-Twisting Itinerant Motion of Electrons
}

\author{
Hiroyasu Koizumi
}

Division of Quantum Condensed Matter Physics, Center for Computational Sciences, University of Tsukuba, Tsukuba 305-8577, Japan; koizumi.hiroyasu.fn@u.tsukuba.ac.jp

Received: 21 February 2020; Accepted: 1 May 2020; Published: 7 May 2020

\begin{abstract}
We show that the Rashba spin-orbit interaction causes spin-twisting itinerant motion of electrons in metals and realizes the quantized cyclotron orbits of conduction electrons without an external magnetic field. From the view point of the Berry connection, the cause of this quantization is the appearance of a non-trivial Berry connection $\mathbf{A}^{\mathrm{fic}}=-\frac{\hbar}{2 e} \nabla \chi(\chi$ is an angular variable with period $2 \pi$ ) that generates $\pi$ flux (in the units of $\hbar=1, e=1, c=1$ ) inside the nodal singularities of the wave function ( $a$ "Dirac string") along the centers of spin-twisting. Since it has been shown in our previous work that the collective mode of $\nabla \chi$ is stabilized by the electron-pairing and generates supercurrent, the $\pi$-flux Dirac string created by the spin-twisting itinerant motion will be stabilized by the electron-pairing and produce supercurrent.
\end{abstract}

Keywords: superconductivity; Dirac string ; Rashba spin-orbit interaction ; spin-twisting itinerant motion

\section{Introduction}

Dirac considered a string of singularities of a wave function with flux through it; he showed that a magnetic monopole should exists at a terminal end of the string [1]. The vortex line in a type II superconductor may be considered as a realization of such an object with a magnetic monopole at a terminal end of it at the surface of the superconductor.

The vortex in the superconductor is explained by the emergence of a vector potential

$$
\mathbf{A}^{\mathrm{fic}}=-\frac{\hbar}{2 e} \nabla \chi
$$

which accompanies the electromagnetic vector potential $\mathbf{A}^{\mathrm{em}}$, where $\hbar$ is Planck's constant divided by $2 \pi,-e$ is the electron charge, and $\chi$ is an angular variable with period $2 \pi$. The sum of the electromagnetic vector potential $\mathbf{A}^{\mathrm{em}}$ and $\mathbf{A}^{\mathrm{fic}}$

$$
\mathbf{A}^{\mathrm{eff}}=\mathbf{A}^{\mathrm{em}}+\mathbf{A}^{\mathrm{fic}}
$$

is an effective gauge invariant vector potential existing in superconductors [2].

The standard theory of superconductivity is the BCS theory [3]. It was originally developed from the energy gap model of Bardeen [4], and identified the cause of the energy gap as the electron pair formation. The BCS theory has been successfully predicted the superconducting transition temperature $T_{c}$, where $T_{c}$ is given as the energy gap formation temperature. The appearance of $\chi$ in $\mathbf{A}^{\mathrm{fic}}$ is due to the use of the particle number non-fixed wave function in the BCS theory; namely, it is attributed to the $U(1)$ gauge symmetry breaking, thus it has been believed that the particle number non-fixed formalism is crucial for a superconductivity theory $[5,6]$. 
Due to the success of the BCS theory, many researchers had thought that superconductivity was a completely solved problem; however, the high temperature superconductivity found in cuprates [7] has proved it is not so. The superconductivity in the cuprate (the cuprate superconductivity) is markedly different from the superconductivity explained by the BCS theory (the BCS superconductivity). Apart from the very high superconducting transition temperature, differences include,

(1) The normal state from which the superconducting state emerges is a doped Mott-insulator [8] although the BCS superconductivity assumes the band metal for the normal state.

(2) Local magnetic correlations in the superconducting state and a close relation between the magnetism and superconductivity have bee observed in the cuprate $[9,10]$, while the magnetism is harmful for the BCS superconductivity.

(3) The superconducting coherence length of the cuprate is in the order of lattice constant (nano-scale) [11], while it is assumed to be much larger than the lattice constant in the BCS superconductivity.

(4) The superconducting transition temperature for the optimally doped cuprate is given by the stabilization temperature of the nano-sized loop currents [12-14], while it is given by the energy gap formation temperature in the BCS superconductivity.

(5) The hole-lattice interaction is very strong and small polarons and bi-polarons are created in the cuprate [15-18], while the BCS superconductivity does not assume such a strong electron-lattice interaction that forms small polarons.

In spite of more than 30 years of research, no widely-accepted theory exists for the mechanim of the cuprate superconductivity. It is very plausible that a drastic departure from the BCS theory is needed for the elucidation of the cuprate superconductivity.

In order to explain the cuprate superconductivity, a new supercurrent generation mechanism where $\mathbf{A}^{\text {fic }}=-\frac{\hbar}{2 e} \nabla \chi$ appears as the Berry connection [19,20] has been put forward [21-24]. In this theory, a macroscopic supercurrent is generated as a collection of spin-vortex-induced loop currents (SVILCs), where the SVILC is a superconducting-coherence-length-sized loop current induced by a spin-vortex (SV) created around each doped hole in the $\mathrm{CuO}_{2}$ plane. It explains a number of experimental results in the cuprate superconductors [25];

(1) Nonzero Kerr rotation in zero-magnetic field after exposed in a strong magnetic field [26].

(2) The change of the sign of the Hall coefficient with temperature change [27].

(3) The suppression of superconductivity in the $x=1 / 8$ static-stripe ordered sample [9].

(4) A large anomalous Nernst signal, including its sign-change with temperature change [28].

(5) The hourglass-shaped magnetic excitation spectrum [9].

(6) Fermi-arc observed in the AEPES [29].

Actually, the new supercurrent generation mechanism does not require the electron-pair formation for the supercurrent generation (this does not mean that the electron pairing is not relevant to the cuprate superconductivity); however, the resulting supercurrent explains the flux quantum $\Phi_{0}=h / 2 e$ and the voltage quantum $V_{0}=h f / 2 e$ ( $f$ is the frequency of the radiation field).

Motivated by the above developments, we have reinvestigated the superfluidity problem in general [30]. Then, we have found that the particle number non-fixed formalism, such as the standard BCS formalism or the Bogoliubov-de Gennes formalism [31,32], can be cast in a particle number fixed formalism if the Berry connection is employed. This indicates that the $U(1)$ gauge symmetry breaking origin of $\mathbf{A}^{\text {fic }}$ may be replaced by the Berry connection origin.

Since the persistent current in topological insulators can be attributed to the Berry connection [33], the Berry connection may be the unified ingredient for persistent current generation in superconductors and topological insulators.

In the present work, we put forward a possible appearance of $\mathrm{A}^{\text {fic }}$ in the BCS superconductor from the view point of the Berry connection origin. In this theory, the nontrivial Berry connection 
arises from the spin-twisting itinerant motion of electrons. Such a motion is realized by the spin-orbit interaction [34]

$$
\frac{e}{2 m^{2} c^{2}} \mathbf{s} \cdot\left[\mathbf{E}^{\mathrm{em}} \times\left(\mathbf{p}+e \mathbf{A}^{\mathrm{em}}\right)\right]
$$

where $\mathbf{s}$ is the electron spin angular momentum, $m$ is electron mass, and $\mathbf{E}^{\mathrm{em}}$ is an electric field. When this interaction affects conduction electrons, it is called the Rashba spin-orbit interaction [35]. Since the internal electric field $\mathbf{E}^{\mathrm{em}}$ exists more or less in any materials, the Rashba interaction exists more or less in any materials. In this work, we consider the case where the Rashba interaction energy is much smaller than the energy gap created by the electron-pairing.

The organization of the present work is following: in Section 2, Bloch electrons under the influence of a magnetic field and the Rashba spin-orbit interaction are investigated. It is shown that the quantization of orbits is realized even without an external magnetic field due to the existence of $\mathbf{A}^{\mathrm{fic}}$ arising from the spin-twisting itinerant motion of electrons. In Section 3, the energy gap for the BCS model is obtained for the case where the electron pairing occurs between $\left(\mathbf{k}, \mathbf{s}_{0}(\mathbf{r})\right)$ and $\left(-\mathbf{k},-\mathbf{s}_{0}(\mathbf{r})\right)$, where $\mathbf{k}$ is the wave vector and $\mathbf{s}_{0}(\mathbf{r})$ is the spin for the electron that depends on the coordinate $\mathbf{r}$ due to the spin-twisting itinerant motion of electrons. In Section 4, the kinetic energy gain by the spin-twisting itinerant motion is investigated. The energy reduction is shown to be optimum when the Berry connection is given by $\mathbf{A}^{\text {fic }}=-\frac{\hbar}{2 e} \nabla \chi$, and the Meissner effect is realized if the nontrivial $\mathbf{A}^{\text {fic ic }}$ stable. In Section 5, the Berry connection from the many-body wave function [30], $\mathbf{A}^{\mathrm{MB}}$, is calculated for the present model. It is shown to be identified as $\mathbf{A}^{\text {fic }}$. According to our previous work [30], $\mathrm{A}^{\mathrm{MB}}$ is stabilized by the electron-pairing, meaning that the $\mathbf{A}^{\text {fic }}$ is stabilized by the electron-pairing; thus, a stable nontrivial $\mathbf{A}^{\text {fic }}$ necessary fo superconductivity is realized. In Section 6, we succinctly summarize part of our previous work that utilizes the particle-number fixed version of the BCS ground state[30], and conclude the present work by discussing implications for it.

\section{Appearance of Spin-Twisting Itinerant Motion of Bloch Electrons under the Influence of the Rashba Spin-Orbit Interaction}

In this section we consider Bloch electrons under the influence of the Rashba interaction and magnetic field. We calculate the quantized energy for them using the method of the periodic-orbit quantization. It is known that the quantized energy obtained in this way gives an accurate energy [36].

In order to obtain the periodic orbit, we use the wave-packet dynamics formalism [37]. In this formalism, the motion of the center of the wave packet corresponds to the classical motion of the electron. The force for the classical motion can be evaluated using the wave packet localized both in the real coordinate space $\mathbf{r}$ and the wave vector space $\mathbf{q}$ under the constraint of the Heisenberg uncertainty condition [38].

Let us consider electrons in a single band and denote its Bloch wave as

$$
\left|\psi_{\mathbf{q}}\right\rangle=e^{i \mathbf{q} \cdot \mathbf{r}}\left|u_{\mathbf{q}}\right\rangle
$$

where $\mathbf{q}$ is the wave vector and $\left|u_{\mathbf{q}}\right\rangle$ is the periodic part of the Bloch wave.

It satisfies the Schrödinger equation,

$$
H_{0}[\mathbf{q}]\left|u_{\mathbf{q}}\right\rangle=\mathcal{E}(\mathbf{q})\left|u_{\mathbf{q}}\right\rangle,
$$

where $H_{0}$ is the zeroth order single-particle Hamiltonian for an electron in a periodic potential.

According to the wave packet dynamics formalism, $H_{0}[\mathbf{q}]$ is modified as

$$
H_{0}[\mathbf{q}] \rightarrow H_{0}\left[\mathbf{q}+\frac{e}{\hbar} \mathbf{A}^{\mathrm{em}}(\mathbf{r})\right]
$$

in the presence of the magnetic field $\mathbf{B}^{\mathrm{em}}=\nabla \times \mathbf{A}^{\mathrm{em}}$. 
Using the Bloch waves, a wave-packet centered at coordinate $\mathbf{r}_{c}$ and central wave vector $\mathbf{q}_{c}$ is constructed as

$$
\left\langle\mathbf{r} \mid\left(\mathbf{q}_{c}, \mathbf{r}_{c}\right)\right\rangle=\int d^{3} q a(\mathbf{q}, t)\left\langle\mathbf{r} \mid \psi_{\mathbf{q}}\right\rangle \Sigma_{1}(\mathbf{r})
$$

where $a(\mathbf{q}, t)$ is a distribution function, and $\Sigma_{1}(\mathbf{r})$ is a spin function. In the present model, we assume the situation where the band is approximated by the extended zone scheme for the nearly free electron model as has been done in the original BCS derivation [3]. Then, the single-band approximation above is adequate. However, in reality, the construction of the wave packet may need many-band Bloch states.

An important point is that we use the spin function that depends on the coordinate $\mathbf{r}$ given by

$$
\Sigma_{1}(\mathbf{r})=e^{-\frac{i}{2} \chi(\mathbf{r})}\left(\begin{array}{c}
e^{-i \frac{1}{2} \xi(\mathbf{r})} \sin \frac{\zeta(\mathbf{r})}{2} \\
e^{\left.i \frac{1}{2} \zeta \mathbf{r}\right)} \cos \frac{\zeta(\mathbf{r})}{2}
\end{array}\right)
$$

where $\zeta(\mathbf{r})$ and $\xi(\mathbf{r})$ are the polar and azimuthal angles of the spin-direction, respectively. This coordinate dependence is necessary to describe the spin-twisting itinerant motion. The expectation value of spin $\mathbf{s}(\mathbf{r})=\left(s_{x}(\mathbf{r}), s_{y}(\mathbf{r}), s_{z}(\mathbf{r})\right)$ is given by

$$
s_{x}(\mathbf{r})=\frac{\hbar}{2} \cos \xi(\mathbf{r}) \sin \zeta(\mathbf{r}), s_{y}(\mathbf{r})=\frac{\hbar}{2} \sin \xi(\mathbf{r}) \sin \zeta(\mathbf{r}), s_{z}=\frac{\hbar}{2} \cos \zeta(\mathbf{r})
$$

In the following argument, the single-valued requirement of the wave function as a function of the coordinate is a crucial condition. This is the postulate adopted by Schrödinger [39], and we impose this condition on the wave packet.

The phase factor $e^{-\frac{i}{2} \chi(\mathbf{r})}$ in $\Sigma_{1}(\mathbf{r})$ is introduced to impose the single-valued condition. Let us consider an example case where the electron performs spin-twisting itinerant motion in which $\xi(\mathbf{r}) \rightarrow \xi(\mathbf{r})+2 \pi$ occurs after a circular transport along a loop in the coordinate space. This leads to the sign-change in $e^{ \pm i \frac{1}{2} \xi(\mathbf{r})}$, and which is compensated by $e^{-\frac{i}{2} \chi(\mathbf{r})}$, for example, this sign change is compensated if $\chi(\mathbf{r}) \rightarrow \chi(\mathbf{r})+2 \pi$ occurs for the same circular transport. The explicit condition for this compensation will be given, later.

The distribution function $a(\mathbf{q}, t)$ satisfies the normalization

$$
\int d^{3} q|a(\mathbf{q}, t)|^{2}=1
$$

and the localization condition in $\mathbf{k}$ space,

$$
\int d^{3} q \mathbf{q}|a(\mathbf{q}, t)|^{2}=\mathbf{q}_{c}
$$

The distribution of $|a(\mathbf{q}, t)|^{2}$ is assumed to be narrow compared with the Brillouin zone size so that $\mathbf{q}_{c}$ can be regarded as the central wave vector of the wave packet.

The wave packet is also localized in $\mathbf{r}$ space around the central position $\mathbf{r}_{c}$,

$$
\mathbf{r}_{c}=\left\langle\left(\mathbf{q}_{c}, \mathbf{r}_{c}\right)|\mathbf{r}|\left(\mathbf{q}_{c}, \mathbf{r}_{c}\right)\right\rangle
$$

The localization in the $\mathbf{q}$ and $\mathbf{r}$ spaces is assumed to satisfy the Heisenberg uncertainty principle [38].

We include the following Rashba interaction term in the Hamiltonian

$$
H_{s o}=\lambda(\mathbf{r}) \cdot \frac{\hbar \sigma}{2} \times\left(\hat{\mathbf{p}}-q \mathbf{A}^{\mathrm{em}}(\mathbf{r})\right),
$$


where $\lambda(\mathbf{r})$ is the spin-orbit coupling vector (its direction is the internal electric field direction), $\hat{\mathbf{p}}=-i \hbar \nabla$ is the momentum operator, and $q=-e$ is electron charge [35].

Let us construct the Lagrangian $L^{\prime}\left(\mathbf{r}_{c}, \dot{\mathbf{r}}_{c}, \mathbf{q}_{c}, \dot{\mathbf{q}}_{c}\right)$ using the time-dependent variational principle [40],

$$
L^{\prime}=\left\langle\left(\mathbf{q}_{c}, \mathbf{r}_{c}\right)\left|i \hbar \frac{\partial}{\partial t}-H\right|\left(\mathbf{q}_{c}, \mathbf{r}_{c}\right)\right\rangle
$$

where $H$ is composed of the Hamiltonian for the band electron that gives the band dispersion $\mathcal{E}\left(\mathbf{q}_{c}+\frac{e}{\hbar} \mathbf{A}_{1}^{\text {eff }}\left(\mathbf{r}_{c}\right)\right)$ and the Rashba interaction $H_{s o}$.

For convenience sake, we introduce another Lagrangian $L$ that is related to $L^{\prime}$ as

$$
L=L^{\prime}-\hbar \frac{d}{d t}\left[\gamma\left(\mathbf{q}_{c}, t\right)-\mathbf{r}_{c} \cdot \mathbf{q}_{c}\right],
$$

where $\gamma$ is the phase of $a(\mathbf{q}, t)=|a(\mathbf{q}, t)| e^{-i \gamma(\mathbf{q}, t)}$.

By following procedures for calculating expectation values for operators by the wave packet [37], $L$ is obtained as

$$
\begin{aligned}
L & =-\mathcal{E}\left(\mathbf{q}_{c}+\frac{e}{\hbar} \mathbf{A}_{1}^{\text {eff }}\left(\mathbf{r}_{c}\right)\right)+\hbar \mathbf{q}_{c} \cdot \dot{\mathbf{r}}_{c}+i \hbar\left\langle u_{\mathbf{q}} \mid \frac{d u_{\mathbf{q}}}{d t}\right\rangle \\
& +\hbar \lambda\left(\mathbf{r}_{c}\right) \cdot\left[\mathbf{s}\left(\mathbf{r}_{\mathbf{c}}\right) \times\left(\mathbf{q}_{c}+\frac{e}{\hbar} \mathbf{A}_{1}^{\text {eff }}\left(\mathbf{r}_{c}\right)\right)\right]
\end{aligned}
$$

where $\mathbf{s}\left(\mathbf{r}_{c}\right)$ is the expectation value of spin for the wave packet centered at $\mathbf{r}_{c}$ given by

$$
\mathbf{s}\left(\mathbf{r}_{c}\right)=\frac{\hbar}{2}\left\langle\left(\mathbf{q}_{c}, \mathbf{r}_{c}\right)|\sigma|\left(\mathbf{q}_{c}, \mathbf{r}_{c}\right)\right\rangle
$$

and

$$
\mathbf{A}_{1}^{\mathrm{eff}}=\mathbf{A}^{\mathrm{em}}+\mathbf{A}_{1}^{\mathrm{fic}}
$$

where $\mathbf{A}_{1}^{\mathrm{fic}}$ is the Berry connection arising from $\Sigma_{1}$ given by

$$
\mathbf{A}_{1}^{\mathrm{fic}}(\mathbf{r})=-i \frac{\hbar}{e} \Sigma_{1}^{\dagger} \nabla \Sigma_{1}=-\frac{\hbar}{2 e} \nabla \chi(\mathbf{r})+\frac{\hbar}{2 e} \nabla \xi(\mathbf{r}) \cos \zeta(\mathbf{r})
$$

We introduce the following wave vector $\mathbf{k}_{c}$,

$$
\mathbf{k}_{c}=\mathbf{q}_{c}+\frac{e}{\hbar} \mathbf{A}_{1}^{\mathrm{eff}}\left(\mathbf{r}_{c}\right)
$$

and change the dynamical variables from $\mathbf{q}_{c}, \dot{\mathbf{q}}_{c}$ to $\mathbf{k}_{c}, \dot{\mathbf{k}}_{c}$ [37].

Then, the Lagrangian with dynamical variables $\mathbf{r}_{c}, \dot{\mathbf{r}}_{c}, \mathbf{k}_{c}$, and $\dot{\mathbf{k}}_{c}$ is given by

$$
\begin{aligned}
& L\left(\mathbf{r}_{c}, \dot{\mathbf{r}}_{c}, \mathbf{k}_{c}, \dot{\mathbf{k}}_{c}\right)=-\mathcal{E}\left(\mathbf{k}_{c}\right)+\hbar \lambda\left(\mathbf{r}_{c}\right) \cdot\left[\mathbf{s}\left(\mathbf{r}_{c}\right) \times \mathbf{k}_{c}\right] \\
+ & \hbar\left[\mathbf{k}_{c}-\frac{e}{\hbar} \mathbf{A}^{\mathrm{eff}}\left(\mathbf{r}_{c}\right)\right] \cdot \dot{\mathbf{r}}_{c}+i \hbar \dot{\mathbf{k}}_{c} \cdot\left\langle u_{\mathbf{q}} \mid \frac{\partial u_{\mathbf{q}}}{\partial \mathbf{q}}\right\rangle_{\mathbf{q}=\mathbf{k}_{c}}
\end{aligned}
$$

Using the above Lagrangian $L$, the following equations of motion are obtained,

$$
\begin{aligned}
\dot{\mathbf{r}}_{c} & =\frac{1}{\hbar} \frac{\partial \mathcal{E}}{\partial \mathbf{k}_{c}}+\lambda\left(\mathbf{r}_{c}\right) \times \mathbf{s}\left(\mathbf{r}_{c}\right)-\dot{\mathbf{k}}_{c} \times \mathbf{\Omega}, \\
\dot{\mathbf{k}}_{c} & =\frac{\partial}{\partial \mathbf{r}_{c}}\left[\lambda\left(\mathbf{r}_{c}\right) \times \mathbf{s}\left(\mathbf{r}_{c}\right) \cdot \mathbf{k}_{c}\right]-\frac{e}{\hbar} \dot{\mathbf{r}}_{c} \times \mathbf{B}^{\mathrm{eff}},
\end{aligned}
$$


where $\Omega$ is the Berry curvature in $\mathbf{k}$ space defined by

$$
\mathbf{\Omega}=i \hbar \nabla_{\mathbf{q}} \times\left\langle u_{\mathbf{q}}\left|\nabla_{\mathbf{q}}\right| u_{\mathbf{q}}\right\rangle
$$

and $\mathbf{B}^{\text {eff }}$ is the effective magnetic field,

$$
\mathbf{B}^{\mathrm{eff}}=\nabla \times \mathbf{A}_{1}^{\mathrm{eff}}
$$

In the following, we consider the case where $\boldsymbol{\Omega}=0$. Then, Equation (22) becomes

$$
\dot{\mathbf{r}}_{c}=\frac{1}{\hbar} \frac{\partial \mathcal{E}\left(\mathbf{k}_{c}\right)}{\partial \mathbf{k}_{c}}+\lambda\left(\mathbf{r}_{c}\right) \times \mathbf{s}\left(\mathbf{r}_{c}\right) .
$$

Using Equations (23) and (26) becomes,

$$
\begin{aligned}
\dot{\mathbf{k}}_{c} & =\frac{\partial}{\partial \mathbf{r}_{c}}\left[\left(\dot{\mathbf{r}}_{c}-\frac{1}{\hbar} \frac{\partial \mathcal{E}\left(\mathbf{k}_{c}\right)}{\partial \mathbf{k}_{c}}\right) \cdot \mathbf{k}_{c}\right]-\frac{e}{\hbar} \dot{\mathbf{r}}_{c} \times \mathbf{B}^{\mathrm{eff}} \\
& =-\frac{e}{\hbar} \dot{\mathbf{r}}_{c} \times \mathbf{B}^{\mathrm{eff}}
\end{aligned}
$$

Equations (26) and (27) indicate that the wave packet exhibits cyclotron motion for the electron in the band with energy

$$
\mathcal{E}(\mathbf{k})+\hbar \lambda(\mathbf{r}) \times \mathbf{s}(\mathbf{r}) \cdot \mathbf{k}
$$

By following the Onsager's argument, let us quantize the cyclotron orbit [41]. From Equation (21), the Bohr-Sommerfeld relation becomes

$$
\oint_{C}\left(\hbar \mathbf{k}_{c}-e \mathbf{A}_{1}^{\mathrm{eff}}\right) \cdot d \mathbf{r}_{c}=2 \pi \hbar\left(n+\frac{1}{2}\right)
$$

where $n$ is an integer and $C$ is the closed loop that corresponds to the section of Fermi surface enclosed by the cyclotron orbit.

Using Equation (27), we have

$$
\begin{aligned}
\oint_{C} \hbar \mathbf{k}_{c} \cdot d \mathbf{r}_{C} & =-e \oint_{C} d \mathbf{r}_{c} \cdot \mathbf{r}_{C} \times \mathbf{B}^{\mathrm{eff}}=e \oint_{C} \mathbf{B}^{\mathrm{eff}} \cdot \mathbf{r}_{C} \times d \mathbf{r}_{C} \\
& =2 e \oint_{C} \mathbf{A}_{1}^{\mathrm{eff}} \cdot d \mathbf{r}_{C}
\end{aligned}
$$

Thus, Equation (29) becomes

$$
e \oint_{C} \mathbf{A}^{\mathrm{em}} \cdot d \mathbf{r}_{C}+e \oint_{C} \mathbf{A}_{1}^{\mathrm{fic}} \cdot d \mathbf{r}_{c}=2 \pi \hbar\left(n+\frac{1}{2}\right)
$$

Note that in the usual quantization condition, $\mathbf{A}_{1}^{\text {fic }}$ is absent.

Now we consider the case where $\mathbf{A}_{1}^{\text {fic }}$ is present. The above quantization condition is satisfied even if the magnetic field is absent. In this case, the first term is zero, and we have

$$
-\oint_{C} \frac{1}{2} \nabla \chi(\mathbf{r}) \cdot d \mathbf{r}_{C}+\oint_{C} \frac{1}{2} \nabla \xi(\mathbf{r}) \cos \zeta(\mathbf{r}) \cdot d \mathbf{r}_{C}=2 \pi\left(n+\frac{1}{2}\right)
$$

which can be satisfied by the following two sets of conditions; one is $\zeta=\pi / 2, w_{C}[\chi]=-1$, and $n=0$; and the other is $\zeta=\pi / 2, w_{C}[\chi]=1$, and $n=-1$, where

$$
w_{C}[\chi]=\frac{1}{2 \pi} \oint_{C} \nabla \chi(\mathbf{r}) \cdot d \mathbf{r}
$$


is the winding number of $\chi$ along loop $C$.

We will show later that the condition $\zeta=\pi / 2$ is achieved by the condition of the kinetic energy gain if the electron-pairing occurs.

The condition $\zeta=\pi / 2$ leads to the following requirements

$$
w_{C}[\chi]+w_{C}[\xi]=\text { even number }
$$

for the single-valued condition for the spin function $\Sigma_{1}$ as a function of the coordinate $\mathbf{r}$. If this condition is satisfied, the product of the phase factors $e^{-\frac{i}{2} \chi(\mathbf{r})}$ and $e^{ \pm i \frac{1}{2} \xi^{\tau}(\mathbf{r})}$ in Equation (8) become single-valued.

The condition $w_{C}[\chi]= \pm 1$ requires that $w_{C}[\xi]$ must be odd, thus, $w_{C}[\xi]$ is not zero. The nonzero value of $w_{C}[\xi]$ means that electrons perform spin-twisting itinerant motion. This indicates that the quantized cyclotron motion may occur without an external magnetic field when the itinerant motion is accompanied by the spin-twisting.

\section{The Pairing Energy Gap}

The results in the previous section indicate that due to the presence of the Rashba interaction, the band energy becomes the one in Equation (28), and Bloch electrons may perform the spin-twisting itinerant motion.

In this section, we consider a modified BCS model where the pairing between single particle states $\left(\mathbf{k}, \mathbf{s}_{0}(\mathbf{r})\right)$ and $\left(-\mathbf{k},-\mathbf{s}_{0}(\mathbf{r})\right)$ occurs, instead between $(\mathbf{k}, \uparrow)$ and $(-\mathbf{k}, \downarrow)$.

Since we use the results of the BCS theory below, let us briefly review it first [3]. The Hamiltonian for the BCS model is given by $H_{\text {kin }}+H_{\text {int }}$, where $H_{\text {kin }}$ is the kinetic energy given by

$$
H_{\text {kin }}=\sum_{\mathbf{k} \sigma} \xi_{0}(\mathbf{k}) c_{\mathbf{k} \sigma}^{\dagger} c_{\mathbf{k} \sigma}
$$

$\xi(\mathbf{k})$ is the energy measured from the Fermi energy $\mu$ given by

$$
\xi_{0}(\mathbf{k})=\mathcal{E}(\mathbf{k})-\mu
$$

and $H_{\text {int }}$ is the interaction energy given by

$$
H_{\text {int }}=\frac{1}{2} \sum_{\mathbf{k} \ell} V_{\mathbf{k} \ell} c_{\mathbf{k} \uparrow}^{\dagger} c_{-\mathbf{k} \downarrow}^{\dagger} c_{-\ell \downarrow} c_{\ell \uparrow} .
$$

The electron pairing occurs between electrons near the Fermi surface due to an attractive $V_{\mathbf{k} \ell}$ that exists in that region. In the BCS model, $V_{\mathbf{k} \ell}$ is nonzero $\left(V_{\mathbf{k} \ell}=-g\right)$ only when $\left|\xi_{0}(\mathbf{k})\right|,\left|\xi_{0}(\ell)\right|<\hbar \omega_{D}$ ( $\omega_{D}$ is the Debye frequency) is satisfied. Then, $\Delta_{\mathbf{k}}$ becomes independent of $\mathbf{k}$, will be expressed as $\Delta$.

The superconducting state is given by the following state vector,

$$
|\mathrm{BCS}\rangle=\prod_{\mathbf{k}}\left(u_{\mathbf{k}}+v_{\mathbf{k}} c_{\mathbf{k} \uparrow}^{\dagger} c_{-\mathbf{k} \downarrow}^{\dagger}\right)|\mathrm{vac}\rangle .
$$

This state exploits the attractive interaction between electron pairs $(\mathbf{k}, \uparrow)$ and $(-\mathbf{k}, \downarrow)$, and the following energy gap equation is obtained,

$$
\Delta=g \sum_{\left|\tilde{\xi}_{0}(\ell)\right|<\hbar \omega_{D}} u_{\ell} v_{\ell}
$$


where $u_{\mathbf{k}}$ and $v_{\mathbf{k}}$ are parameters given using $\Delta$ and $\xi(\mathbf{k})_{0}$ as

$$
u_{\mathbf{k}}=\frac{1}{\sqrt{2}}\left(1+\frac{\xi_{0}(\mathbf{k})}{\sqrt{\xi_{0}^{2}(\mathbf{k})+\Delta^{2}}}\right)^{1 / 2}
$$

and

$$
v_{\mathbf{k}}=\frac{1}{\sqrt{2}}\left(1-\frac{\xi_{0}(\mathbf{k})}{\sqrt{\xi_{0}^{2}(\mathbf{k})+\Delta^{2}}}\right)^{1 / 2}
$$

respectively.

The total energy by the formation of the energy gap is given by

$$
E_{\mathrm{s}}^{\mathrm{BCS}}=E_{\mathrm{n}}^{\mathrm{BCS}}-\frac{1}{2} N(\mu) \Delta^{2}
$$

where $E_{\mathrm{n}}^{\mathrm{BCS}}$ is the normal state energy, and $N(\mu)$ is the density of states at the Fermi energy $\mu$ [3].

Now, consider the pairing of $\left(\mathbf{k}, \mathbf{s}_{0}(\mathbf{r})\right)$ and $\left(-\mathbf{k},-\mathbf{s}_{0}(\mathbf{r})\right)$, and also $\left(\mathbf{k},-\mathbf{s}_{0}(\mathbf{r})\right)$ and $\left(-\mathbf{k}, \mathbf{s}_{0}(\mathbf{r})\right)$. We divide the system into coarse-grained cells of volume 1 to take into account the coordinate dependence of the band energy in Equation (28), assuming that its coordinate dependence is very slow. Then, the ground state in the cell with the central position $\mathbf{r}$ is given by

$$
\left.|\operatorname{BCS}(\mathbf{r})\rangle=\prod_{\mathbf{k}}\left(u_{\mathbf{k}}(\mathbf{r})+v_{\mathbf{k}}(\mathbf{r}) \mathcal{C}_{\mathbf{k} \uparrow}^{\dagger} \mathcal{C}_{-\mathbf{k} \downarrow}^{\dagger}\right) \mid \text { vac }\right\rangle
$$

where $u_{ \pm}(\mathbf{k}, \mathbf{r})$ and $v_{ \pm}(\mathbf{k}, \mathbf{r})$ are given by

$$
\begin{aligned}
& u_{ \pm}\left(\mathbf{k}_{c}, \mathbf{r}\right)=\frac{1}{\sqrt{2}}\left(1+\frac{\xi_{ \pm}(\mathbf{k}, \mathbf{r})}{\sqrt{\xi_{ \pm}^{2}(\mathbf{k}, \mathbf{r})+\Delta^{2}(\mathbf{r})}}\right)^{1 / 2}, \\
& v_{ \pm}(\mathbf{k}, \mathbf{r})=\frac{1}{\sqrt{2}}\left(1-\frac{\xi_{ \pm}(\mathbf{k}, \mathbf{r})}{\sqrt{\xi_{ \pm}^{2}(\mathbf{k}, \mathbf{r})+\Delta^{2}(\mathbf{r})}}\right)^{1 / 2}
\end{aligned}
$$

with

$$
\xi_{ \pm}(\mathbf{k}, \mathbf{r})=\mathcal{E}_{ \pm}(\mathbf{k})-\mu, \quad \mathcal{E}_{ \pm}(\mathbf{k}, \mathbf{r})=\mathcal{E}(\mathbf{k}) \pm \hbar \lambda(\mathbf{r}) \times \mathbf{k} \cdot \mathbf{s}_{0}(\mathbf{r})
$$

Then, the gap function $\Delta(\mathbf{r})$ is the solution of the gap equation given by

$$
\begin{aligned}
\Delta(\mathbf{r}) & =\frac{g}{2} \sum_{\left|\xi_{0}(\ell)\right|<\hbar \omega_{D}}\left\{u_{+}(\ell, \mathbf{r}) v_{+}(\ell, \mathbf{r})+u_{-}(\ell, \mathbf{r}) v_{-}\left(\ell_{c}, \mathbf{r}\right)\right\} \\
& =\frac{g \Delta(\mathbf{r})}{4} \sum_{\left|\xi_{0}(\ell)\right|<\hbar \omega_{D}}\left\{\frac{1}{\sqrt{\xi_{+}^{2}(\mathbf{k}, \mathbf{r})+\Delta^{2}\left(\mathbf{r}_{c}\right)}}+\frac{1}{\sqrt{\xi_{-}^{2}(\mathbf{k}, \mathbf{r})+\Delta^{2}(\mathbf{r})}}\right\} \\
& \approx \frac{g \Delta(\mathbf{r})}{4} \sum_{\left|\xi_{0}(\ell)\right|<\hbar \omega_{D}}\left\{\frac{2}{\sqrt{\xi_{0}^{2}(\mathbf{k}, \mathbf{r})+\Delta^{2}(\mathbf{r})}}-\frac{\lambda^{2}}{\left[\xi_{0}^{2}(\mathbf{k}, \mathbf{r})+\Delta^{2}(\mathbf{r})\right]^{3 / 2}}\right\} \\
& \approx \frac{g \Delta(\mathbf{r}) N(\mu ; \mathbf{r})}{4} \int_{-\hbar \omega_{D}}^{\hbar \epsilon}\left\{\frac{2}{\sqrt{\epsilon^{2}+\Delta^{2}(\mathbf{r})}}-\frac{\lambda^{2}}{\left[\epsilon^{2}+\Delta^{2}(\mathbf{r})\right]^{3 / 2}}\right\} \\
& \approx g \Delta(\mathbf{r}) N(\mu ; \mathbf{r})\left\{\log \frac{2 \hbar \omega_{D}}{\Delta(\mathbf{r})}-\frac{\lambda^{2}}{\Delta^{2}(\mathbf{r})}\right\}
\end{aligned}
$$


where $N(\mu ; \mathbf{r})$ is the density of states at the Fermi energy in the coarse grained cell with the central position $\mathbf{r}$, and $\lambda=|\lambda|$.

From the above relation, we obtain the following,

$$
\Delta(\mathbf{r}) \approx 2 \hbar \omega_{D} \exp \left(-\frac{1}{g N(\mu ; \mathbf{r})}-\frac{\lambda^{2}}{\Delta_{0}^{2}}\right) ; \quad \Delta_{0}(\mathbf{r})=2 \hbar \omega_{D} \exp \left(-\frac{1}{g N(\mu ; \mathbf{r})}\right)
$$

where $\Delta_{0}$ is the gap value without the spin-orbit interaction; here, it is assumed that $\hbar \omega_{D} \gg \Delta$ holds. The gap $\Delta$ is reduced by the spin-orbit interaction. However, if the spin-orbit interaction parameter $\lambda$ is significantly smaller that $\Delta_{0}$, the gap is almost the same as the original one. In the following we assume such a case.

\section{Kinetic Energy Gain by the Spin-Twisting Itinerant Motion}

In this section, we consider the appearance of $\mathbf{A}^{\text {fic }}=-\frac{\hbar}{2 e} \nabla \chi$ from the view point of the kinetic energy gain.

We consider the case where the pair $\left(\mathbf{k}, \mathbf{s}_{0}(\mathbf{r})\right)$ and $\left(-\mathbf{k},-\mathbf{s}_{0}(\mathbf{r})\right)$, and another pair $\left(\mathbf{k},-\mathbf{s}_{0}(\mathbf{r})\right)$ and $\left(-\mathbf{k}, \mathbf{s}_{0}(\mathbf{r})\right)$, are both occupied. We assume that $\mathbf{s}_{0}(\mathbf{r})$ for the first pair arises from the spin function $\Sigma_{1}$ in Equation (8), and $-\mathbf{s}_{0}(\mathbf{r})$ for the second pair arises from the spin function $\Sigma_{2}$ given by

$$
\Sigma_{2}(\mathbf{r})=e^{-\frac{i}{2} \chi(\mathbf{r})}\left(\begin{array}{c}
i e^{-i \frac{1}{2} \xi(\mathbf{r})} \cos \frac{\zeta(\mathbf{r})}{2} \\
-i e^{\left.i \frac{i}{2} \xi \mathbf{r}\right)} \sin \frac{\zeta(\mathbf{r})}{2}
\end{array}\right)
$$

Note that $\Sigma_{1}$ and $\Sigma_{2}$ are orthogonal.

The fictitious vector potential $\mathbf{A}_{2}^{\text {fic }}(\mathbf{r})$ from $\Sigma_{2}$ is calculated as

$$
\mathbf{A}_{2}^{\mathrm{fic}}(\mathbf{r})=-i \frac{\hbar}{e} \Sigma_{2}^{\dagger} \nabla \Sigma_{2}=-\frac{\hbar}{2 e} \nabla \chi(\mathbf{r})-\frac{\hbar}{2 e} \nabla \xi(\mathbf{r}) \cos \zeta(\mathbf{r})
$$

and the effective vector potential is given by

$$
\mathbf{A}_{2}^{\text {eff }}=\mathbf{A}^{\mathrm{em}}+\mathbf{A}_{2}^{\text {fic }}
$$

The single-particle energy for the pair $\left(\mathbf{k}, \mathbf{s}_{0}(\mathbf{r})\right)$ and $\left(-\mathbf{k},-\mathbf{s}_{0}(\mathbf{r})\right)$ is $\mathcal{E}_{+}(\mathbf{k}, \mathbf{r})$, and that for the pair $\left(\mathbf{k},-\mathbf{s}_{0}(\mathbf{r})\right)$ and $\left(-\mathbf{k}, \mathbf{s}_{0}(\mathbf{r})\right)$ is $\mathcal{E}_{-}(\mathbf{k}, \mathbf{r})$ in Equation (46).

Then, the kinetic energy for the cell at $r$ is given by

$$
2 \sum_{\mathcal{E}_{-}(\mathbf{k}, \mathbf{r})<\mu} \mathcal{E}_{-}(\mathbf{k}, \mathbf{r})+2 \sum_{\mathcal{E}_{+}(\mathbf{k}, \mathbf{r})<\mu} \mathcal{E}_{+}(\mathbf{k}, \mathbf{r})
$$

For simplicity, we approximate the above energy using the Fermi distribution functions $f(\epsilon)=$ $\left(1+e^{\epsilon / k_{B} T}\right)^{-1}\left(k_{B}\right.$ is Boltzmann's constant) and density of states $N(\epsilon ; \mathbf{r})$ as

$$
\begin{aligned}
& \int \frac{N(\epsilon ; \mathbf{r})}{2}\left\{\left[\epsilon+\hbar \lambda(\mathbf{r}) \times \mathbf{k}_{c} \cdot \mathbf{s}_{0}(\mathbf{r})\right] f\left(\epsilon+\hbar \lambda(\mathbf{r}) \times \mathbf{k}_{c} \cdot \mathbf{s}_{0}(\mathbf{r})\right)\right. \\
+ & {\left.\left[\epsilon-\hbar \lambda(\mathbf{r}) \times \mathbf{k}_{c} \cdot \mathbf{s}_{0}(\mathbf{r})\right] f\left(\epsilon-\hbar \lambda(\mathbf{r}) \times \mathbf{k}_{c} \cdot \mathbf{s}_{0}(\mathbf{r})\right)\right\} d \epsilon } \\
\approx & \int \frac{N(\epsilon ; \mathbf{r})}{2}\left\{\epsilon\left[f\left(\epsilon+\hbar \lambda(\mathbf{r}) \times \mathbf{k} \cdot \mathbf{s}_{0}(\mathbf{r})\right)+f\left(\epsilon-\hbar \lambda(\mathbf{r}) \times \mathbf{k} \cdot \mathbf{s}_{0}(\mathbf{r})\right)\right]\right. \\
+ & \left.\hbar \lambda(\mathbf{r}) \times \mathbf{k} \cdot \mathbf{s}_{0}(\mathbf{r})\left[f\left(\epsilon+\hbar \lambda(\mathbf{r}) \times \mathbf{k} \cdot \mathbf{s}_{0}(\mathbf{r})\right)-f\left(\epsilon-\hbar \lambda(\mathbf{r}) \times \mathbf{k} \cdot \mathbf{s}_{0}(\mathbf{r})\right)\right]\right\} d \epsilon \\
\approx & \int \frac{N(\epsilon ; \mathbf{r})}{2}\left\{2 \epsilon f(\epsilon)+2\left|\hbar \lambda(\mathbf{r}) \times \mathbf{k} \cdot \mathbf{s}_{0}(\mathbf{r})\right|^{2} \frac{\partial f(\epsilon)}{\partial \epsilon}\right\} d \epsilon
\end{aligned}
$$


At temperature $T=0, \frac{\partial f(\epsilon)}{\partial \epsilon}=-\delta(\epsilon)$; thus, we have

$$
\int d \epsilon N(\epsilon ; \mathbf{r}) \epsilon f(\epsilon) d \epsilon-N(\mu ; \mathbf{r})\left|\hbar \lambda(\mathbf{r}) \times \mathbf{k}_{c} \cdot \mathbf{s}_{0}(\mathbf{r})\right|^{2}
$$

The first term in Equation (54) may be approximated as

$$
\frac{1}{2} \sum_{\mathcal{E}(\mathbf{q})<\mu, i=1,2} \frac{\hbar^{2}}{2 m}\left[\mathbf{q}+\frac{e}{\hbar} \mathbf{A}_{i}^{\text {eff }}\right]^{2} \approx \sum_{\mathcal{E}(\mathbf{q})<\mu} \frac{\hbar^{2}}{2 m} \mathbf{q}^{2}+\frac{e^{2} \rho(\mathbf{r})}{4 m}\left(\left|\mathbf{A}_{1}^{\text {eff }}\right|^{2}+\left|\mathbf{A}_{2}^{\text {eff }}\right|^{2}\right)
$$

assuming that the term linear in $\mathbf{q}$ cancels out due to the time-reversal and/or inversion symmetry. Here $\rho$ is the number density of electrons (later, we consider it as the number density of electrons participating in the collective mode $\nabla \chi$ ).

The second term in Equation (54) may be approximated as

$$
\begin{aligned}
& -\frac{1}{2} \sum_{\mathcal{E}(\mathbf{q})=\mu, j=1,2}\left|\hbar \lambda(\mathbf{r}) \times\left[\mathbf{q}+\frac{e}{\hbar} \mathbf{A}_{j}^{\mathrm{eff}}\right] \cdot \mathbf{s}_{0}(\mathbf{r})\right|^{2} \\
\approx & -\hbar^{2} \sum_{\mathcal{E}(\mathbf{q})=\mu}\left|\lambda(\mathbf{r}) \times \mathbf{q} \cdot \mathbf{s}_{0}(\mathbf{r})\right|^{2}-\frac{1}{2} \sum_{j=1,2} e^{2} N(\mu ; \mathbf{r})\left|\lambda(\mathbf{r}) \times \mathbf{s}_{0}(\mathbf{r}) \cdot \mathbf{A}_{j}^{\mathrm{eff}}\right|^{2}
\end{aligned}
$$

assuming that the term linear in $\mathbf{q}$ cancels out.

From the condition for minimizing the kinetic energy, $\mathbf{s}_{0}$ is chosen to satisfy

$$
\lambda(\mathbf{r}) \times \mathbf{s}_{0}(\mathbf{r}) \| \mathbf{A}_{1}^{\mathrm{eff}}(\mathbf{r}) \text { and } \mathbf{A}_{2}^{\mathrm{eff}}(\mathbf{r})
$$

We assume $\lambda(\mathbf{r})$ in the coarse-grained cell at $\mathbf{r}$ to be uniform in the $z$-direction; then, the optimal $\mathbf{s}_{0}(\mathbf{r})$ that satisfies the above condition lies in the $x y$ plane. Thus, $\zeta$ in $\mathbf{A}_{1}^{\text {eff }}(\mathbf{r})$ and $\mathbf{A}_{2}^{\text {eff }}(\mathbf{r})$ is taken to be $\zeta=\pi / 2$, yielding common $\mathbf{A}^{\text {fic }}(\mathbf{r})=-\frac{\hbar}{2 e} \nabla \chi$ for $\mathbf{A}_{1}^{\text {fic }}(\mathbf{r})$ and $\mathbf{A}_{2}^{\text {fic }}(\mathbf{r})$. As a consequence, we have the common effective potential given by

$$
\mathbf{A}^{\mathrm{eff}}=\mathbf{A}^{\mathrm{em}}-\frac{\hbar}{2 e} \nabla \chi=\mathbf{A}^{\mathrm{em}}+\mathbf{A}^{\mathrm{fic}}
$$

for $\mathbf{A}_{1}^{\text {eff }}(\mathbf{r})$ and $\mathbf{A}_{2}^{\text {eff }}(\mathbf{r})$.

The kinetic energy increase given by the appearance of $\mathbf{A}^{\text {eff }}$ in Equation (55) is calculated as

$$
\int d^{3} r \frac{e^{2} \rho(\mathbf{r})}{2 m}\left|\mathbf{A}^{\text {eff }}\right|^{2}
$$

This indicates that the optimum $\mathbf{A}^{\mathrm{fic}}$ is the one that gives $\mathbf{A}^{\mathrm{eff}}=0$ if this choice is possible. If we adopt $\mathbf{A}^{\mathrm{em}}=0$ when a magnetic field is zero, the condition yields $\mathbf{A}^{\text {fic }}=0$, i.e., the absence of the spin-twisting itinerant motion. When $\mathbf{A}^{\mathrm{em}} \neq 0$, however, the optimal $\mathbf{A}^{\mathrm{fic}}$ will be the one for the presence of the spin-twisting itinerant motion.

From the kinetic energy, we can calculate the current density as

$$
\mathbf{j}_{\text {tot }}(\mathbf{r})=-e^{2}\left[\frac{\rho(\mathbf{r})}{m}-N(\mu ; \mathbf{r})\left|\lambda(\mathbf{r}) \times \mathbf{s}_{0}(\mathbf{r})\right|^{2}\right] \mathbf{A}^{\mathrm{eff}}(\mathbf{r})
$$

This is the London equation, and the system should exhibit the Meissner effect. Thus, $\mathbf{A}^{\mathrm{eff}}=0$ is realized in the bulk. If the system is a ring-shaped, it will lead to the quantization of magnetic flux in the units $\frac{h}{2 e}$. The Equation (56) indicates the occurrence of the energy reduction in the order of $\lambda^{2}$ if the surface energy is negligible compared to the bulk energy. In other words, when a magnetic field is applied the spin-twisting itinerant motion occurs, and gives rise to $\mathbf{A}^{\text {fic }}$ that causes the Meissner effect and the flux quantization in $\frac{h}{2 e}$. 


\section{Berry Connection for Many-Body Wave Functions and $A^{\text {fic }}$}

We consider $\mathbf{A}^{\text {fic }}$ from the view point of the Berry connection for the many-body wave functions (dented as $\mathbf{A}^{\mathrm{MB}}$ ) introduced in our previous work [30] in this section.

Let us denote the wave function of a system with $N$ electrons as

$$
\Psi\left(\mathbf{x}_{1}, \cdots, \mathbf{x}_{N}, t\right)
$$

where $\mathbf{x}_{j}=\left(\mathbf{r}_{j}, s_{j}\right)$ denotes the coordinate $\mathbf{r}_{j}$ and spin $s_{j}$ of the $j$ th electron.

The Berry connection [19] associated with this wave function is called the "Berry connection for many-body wave function" [30]. In order to calculate this Berry connection, we first prepare the parameterized wave function $\left|n_{\Psi}(\mathbf{r})\right\rangle$ with the parameter $\mathbf{r}$,

$$
\left\langle s, \mathbf{x}_{2}, \cdots, \mathbf{x}_{N} \mid n_{\Psi}(\mathbf{r}, t)\right\rangle=\frac{\Psi\left(\mathbf{r} s, \mathbf{x}_{2}, \cdots, \mathbf{x}_{N}, t\right)}{|C(\mathbf{r}, t)|^{\frac{1}{2}}}
$$

where $|C(\mathbf{r}, t)|$ is the normalization constant given by

$$
|C(\mathbf{r}, t)|=\int d s d \mathbf{x}_{2} \cdots d \mathbf{r}_{N} \Psi\left(\mathbf{r} s, \mathbf{x}_{2}, \cdots\right) \Psi^{*}\left(\mathbf{x} s, \mathbf{x}_{2}, \cdots\right)
$$

Using $\left|n_{\Psi}\right\rangle$, the Berry connection for many-body wave function is given by

$$
\mathbf{A}^{\mathrm{MB}}(\mathbf{r}, t)=-i\left\langle n_{\Psi}(\mathbf{r}, t)\left|\nabla_{\mathbf{r}}\right| n_{\Psi}(\mathbf{r}, t)\right\rangle
$$

Here, $\mathbf{r}$ is regarded as the parameter [19].

When the origin of $\mathrm{A}^{\mathrm{MB}}$ is not the ordinary magnetic field one, i.e.,

$$
\nabla \times \mathbf{A}^{\mathrm{MB}}=0
$$

it can be written in the pure gauge form,

$$
\mathbf{A}^{\mathrm{MB}}=\nabla \theta
$$

where $\theta$ is a function which may be multi-valued.

Let us consider the case where $\Psi$ is given as a Slater determinant of spin-orbitals $\phi_{1,1}(\mathbf{r}) \Sigma_{1}(\mathbf{r}), \phi_{1,2}(\mathbf{r}) \Sigma_{2}(\mathbf{r}), \ldots, \phi_{\frac{N}{2}, 1}(\mathbf{r}) \Sigma_{1}(\mathbf{r})$, and $\phi_{\frac{N}{2}, 2}(\mathbf{r}) \Sigma_{2}(\mathbf{r})$, where $\phi_{j, 1}(\mathbf{r})$ and $\phi_{j, 2}(\mathbf{r})$ are time-reversal partners and $N$ is assumed to be even.

Then, $\mathrm{A}^{\mathrm{MB}}$ is calculated as

$$
\begin{aligned}
\mathbf{A}^{\mathrm{MB}} & =\operatorname{Im} \frac{\sum_{j=1}^{\frac{N}{2}}\left[\phi_{j, 1}^{*}(\mathbf{r}) \Sigma_{1}^{\dagger}(\mathbf{r}) \nabla \phi_{j, 1}(\mathbf{r}) \Sigma_{1}(\mathbf{r})+\phi_{j, 2}^{*}(\mathbf{r}) \Sigma_{2}^{\dagger}(\mathbf{r}) \nabla \phi_{j, 2}(\mathbf{r}) \Sigma_{2}(\mathbf{r})\right]}{\sum_{j=1}^{\frac{N}{2}}\left[\phi_{j, 1}^{*}(\mathbf{r}) \phi_{j, 1}(\mathbf{r})+\phi_{j, 2}^{*}(\mathbf{r}) \phi_{j, 2}(\mathbf{r})\right]} \\
& =\frac{e}{\hbar} \frac{\mathbf{A}_{1}^{\mathrm{fic}} \sum_{j=1}^{\frac{N}{2}} \phi_{\phi, 1}^{*}(\mathbf{r}) \phi_{j, 1}(\mathbf{r})+\mathbf{A}_{2}^{\mathrm{fic}} \sum_{j=1}^{\frac{N}{2}} \phi_{j, 2}^{*}(\mathbf{r}) \phi_{j, 2}(\mathbf{r})}{\sum_{j=1}^{\frac{N}{2}}\left[\phi_{j, 1}^{*}(\mathbf{r}) \phi_{j, 1}(\mathbf{r})+\phi_{j, 2}^{*}(\mathbf{r}) \phi_{j, 2}(\mathbf{r})\right]}
\end{aligned}
$$

where "Im" indicates the imaginary part, and the fact that $\sum_{j=1}^{\frac{N}{2}}\left[\phi_{j, 1}^{*}(\mathbf{r}) \nabla \phi_{j, 1}(\mathbf{r})+\phi_{j, 2}^{*}(\mathbf{r}) \nabla \phi_{j, 2}(\mathbf{r})\right]$ is real (due to the fact that $\phi_{j, 1}(\mathbf{r})$ and $\phi_{j, 2}(\mathbf{r})$ are time-reversal partners) is used.

As is shown in the previous sections, optimal $\mathbf{A}_{1}^{\mathrm{fic}}$ and $\mathbf{A}_{2}^{\mathrm{fic}}$ are given by $\mathbf{A}_{1}^{\mathrm{fic}}=\mathbf{A}_{2}^{\mathrm{fic}}=\mathbf{A}^{\mathrm{fic}}=$ $-\frac{\hbar}{2 e} \nabla \chi$. In this case, we have

$$
\mathbf{A}^{\mathrm{MB}}=\frac{e}{\hbar} \mathbf{A}^{\mathrm{fic}}=-\frac{1}{2} \nabla \chi
$$


thus $\mathbf{A}^{\text {fic }}$ is identified as $\mathbf{A}^{\mathrm{MB}}$ with factor $\frac{\hbar}{e}$. We may identify $\theta$ as $-\chi / 2$.

The kinetic energy part of the Hamiltonian is given by

$$
K_{0}=\frac{1}{2 m} \sum_{j=1}^{N}\left(\frac{\hbar}{i} \nabla_{j}\right)^{2}
$$

where $m$ is the electron mass and $\nabla_{j}$ is the gradient operator with respect to the $j$ th electron coordinate $\mathbf{r}_{j}$.

Using $\Psi$ and $\mathrm{A}^{\mathrm{MB}}$, we can construct a currentless wave function $\Psi_{0}$ for the current operator associated with $K_{0}$

$$
\Psi_{0}\left(\mathbf{x}_{1}, \cdots, \mathbf{x}_{N}, t\right)=\Psi\left(\mathbf{x}_{1}, \cdots, \mathbf{x}_{N}, t\right) \exp \left(-i \sum_{j=1}^{N} \int_{0}^{\mathbf{r}_{j}} \mathbf{A}^{\mathrm{MB}}\left(\mathbf{r}^{\prime}, t\right) \cdot d \mathbf{r}^{\prime}\right)
$$

Reversely, $\Psi\left(\mathbf{x}_{1}, \cdots, \mathbf{x}_{N}, t\right)$ is expressed as

$$
\Psi\left(\mathbf{x}_{1}, \cdots, \mathbf{x}_{N}, t\right)=\Psi_{0}\left(\mathbf{x}_{1}, \cdots, \mathbf{x}_{N}, t\right) \exp \left(-\frac{i}{2} \sum_{j=1}^{N} \chi\left(\mathbf{r}_{j}, t\right)\right)
$$

using the currentless wave function $\Psi_{0}$.

Due to the spin-twisting the winding number of $\chi$ is non-zero, thus, a line of singularities exist within the loop around which the winding number is non-zero. The flux threaded through the line of singularities can be calculated using $\mathbf{A}^{\mathrm{fic}}$, and yields $\pi(\bmod 2 \pi)$; thus, the line of singularities is the $\pi$-flux Dirac string. The wave function in Equation (71) indicates that a collective mode described by $\exp \left(-\frac{i}{2} \sum_{j=1}^{N} \chi\left(\mathbf{r}_{j}, t\right)\right)$ produces the persistent current, and it arises from the Dirac string.

In the present formalism, the superconducting state is the one with nontrivial $\mathbf{A}^{\text {fic }}$. It plays dual roles; it is a Berry connection that enables the comparison of the phase of the wave function at different spatial points and gives rise to the macroscopic quantum interference effects. At the same time, it is the collective mode $\nabla \chi$ of electrons with a long range order of the average momentum [42].

\section{Concluding Remarks}

In the present work, we have shown that the spin-twisting itinerant motion occurs for the conduction electrons of metals due to the Rashba spin-orbit interaction, and it generates $\mathbf{A}^{\mathrm{fic}}$. When the energy gap formation by electron pairing described by the BCS theory occurs, $\mathrm{A}^{\mathrm{fic}}$ is stabilized, and the superconducting state is realized.

The stabilization of $\mathbf{A}^{\text {fic }}$ by the electron-pairing is explained in our previous work [30]. The ground state with the total number of particles $N$ at a coarse-grained cell of volume 1 with the central position $\mathbf{r}$ is given by

$$
|\operatorname{Gnd}(\mathbf{r} ; N)\rangle=\prod_{\mathbf{k}}\left(u_{\mathbf{k}}(\mathbf{r})+v_{\mathbf{k}}(\mathbf{r}) c_{\mathbf{k} \uparrow}^{\dagger} c_{-\mathbf{k} \downarrow}^{\dagger} e^{-i \hat{\chi}(\mathbf{r})}\right)|\operatorname{Cnd}(N)\rangle
$$

where $e^{-i \hat{\chi}(\mathbf{r})}$ is the number changing operator that satisfies

$$
e^{-i \hat{\chi}(\mathbf{r})}|\operatorname{Cnd}(N)\rangle=e^{-i \chi(\mathbf{r})}|\operatorname{Cnd}(N-2)\rangle
$$

and $|\operatorname{Cnd}(N)\rangle$ is the state vector for the condensate which is related to the wave function $\Psi_{0}$ in Equation (70) by

$$
\Psi_{0}\left(\mathbf{x}_{1}, \cdots, \mathbf{x}_{N}, t\right)=\left\langle\mathbf{x}_{1}, \cdots, \mathbf{x}_{N} \mid \operatorname{Cnd}(N)\right\rangle
$$


The number changing operator $e^{-i \hat{\chi}(\mathbf{r})}$ changes the number in the condensate $|\operatorname{Cnd}(N)\rangle$ by two, and also adds the phase factor $e^{-i \chi(\mathbf{r})}[30]$.

The ground state in the new theory $|\operatorname{Gnd}(\mathbf{r} ; N)\rangle$ corresponds to the BCS ground state with the phase factor $e^{-i \chi(\mathbf{r})}$ given by

$$
|\mathrm{BCS}(\mathbf{r} ; \chi)\rangle=\prod_{\mathbf{k}}\left(u_{\mathbf{k}}(\mathbf{r})+v_{\mathbf{k}}(\mathbf{r}) c_{\mathbf{k} \uparrow}^{\dagger} c_{-\mathbf{k} \downarrow}^{\dagger} e^{-i \chi(\mathbf{r})}\right)|\mathrm{vac}\rangle .
$$

Note that the BCS ground state here is not the original one, but the one that varies slowly with coordinate $\mathbf{r}$ due to the coordinate dependence of the parameters, $u_{\mathbf{k}}(\mathbf{r}), v_{\mathbf{k}}(\mathbf{r})$, and $\chi(\mathbf{r})$. This coordinate dependence is necessary to have nontrivial $\mathbf{A}^{\text {fic }}$. A more powerful method to deal with this coordinate dependence is the Bogoliubov-de Gennes method [31,32]; the corresponding version for $|\operatorname{Gnd}(\mathbf{r} ; N)\rangle$ will be found in our previous work [30].

The two ground states, $|\operatorname{Gnd}(\mathbf{r} ; N)\rangle$ and $|\operatorname{BCS}(\mathbf{r} ; \chi)\rangle$, look very similar, and the mathematical structures arising from them are also very similar. However, there exists a serious difference in concerning the ac Josephson effect. This difference indicates the new formalism is more in accordance with the observed ac Josephson effect $[21,24]$. Let us explain this point below.

A serious misfit was found in the recent re-derivation of the ac Josephson effect [43]. The boundary condition considered by Josephson and the one employed in the experiment are actually different; if the experimental boundary condition is employed with taking into account the gauge invariance, the observed Josephson relation indicates that the charge on the particle is $q=-e$ not $q=-2 e[21,24]$. The new formalism can explain the experimentally observed ac Josephson effect with $q=-e$ since the role of the electron pairing is the stabilization of $\mathbf{A}^{\text {fic }}$ and the charge $q=-e$ supercurrent flow is possible.

The charge $q=-e$ supercurrent flow through the Josephson junction is explained in the new formalism as follows. We first introduce the following particle number conserving Bogoliubov transformation [30],

$$
\begin{aligned}
& c_{i \sigma}=\sum_{n}\left[u_{i}^{n} \gamma_{n \sigma}-\sigma\left(v_{i}^{n}\right)^{*} \gamma_{n-\sigma}^{\dagger}\right] e^{-\frac{i}{2} \hat{\chi}_{i}} \\
& c_{i \sigma}^{\dagger}=\sum_{n}\left[\left(u_{i \sigma}^{n}\right)^{*} \gamma_{n \sigma}^{\dagger}-\sigma v_{i}^{n} \gamma_{n-\sigma}\right] e^{\frac{i}{2} \hat{x}_{i}}
\end{aligned}
$$

where $\sigma$ is the spin, +1 for $\uparrow$, and -1 for $\downarrow ; c_{i \sigma}$ and $c_{i \sigma}^{\dagger}$ are annihilation and creation operators for electrons with spin $\sigma$ at the $i$ th site; $\gamma_{n \sigma}$ and $\gamma_{n \sigma}^{\dagger}$ are the the particle number conserving Bogoliubov operators; $u_{i}^{n}$ and $v_{i}^{n}$ are parameters obtained by solving the Bogoliubov-de Gennes equations; $e^{-\frac{i}{2} \hat{\chi}_{i}}$ is the number changing operator that reduces the number of particle in the condensate by one at the $i$ th site, thereby, introduces a phase factor $e^{-\frac{i}{2} \chi_{i}}$.

We use the following electron transfer Hamiltonian for the Josephson junction,

$$
H_{L R}=-\sum_{\sigma} T_{L R}\left(c_{L \sigma}^{\dagger} c_{R \sigma}+c_{R \sigma}^{\dagger} c_{L \sigma}\right)
$$

Labels" $L$ " and " $R$ " refer to quantities for the left superconductor $S_{L}$ and right superconductor $S_{R}$, respectively.

Using the Boboliubov transformation in Equation (76) and including the electromagnetic field by the Peierls substitution, $H_{L R}$ is rewritten as

$$
\begin{aligned}
& H_{L R}=-T_{L R} e^{\frac{i}{2}\left(\hat{\chi}_{L}-\hat{\chi}_{R}\right)} e^{-i \frac{e}{\hbar} \int_{R}^{L} d \mathbf{r} \cdot \mathbf{A}^{\mathrm{em}}} \sum_{n, m}\left[\left(\left(u_{L}^{n}\right)^{*} \gamma_{n \downarrow}^{\dagger}+v_{L}^{n} \gamma_{n \uparrow}\right)\left(u_{R}^{m} \gamma_{m \downarrow}+\left(v_{R}^{m}\right)^{*} \gamma_{m \uparrow}^{\dagger}\right)\right. \\
& \left.+\left(\left(u_{L}^{n}\right)^{*} \gamma_{n \uparrow}^{\dagger}-v_{L}^{n} \gamma_{n \downarrow}\right)\left(u_{R}^{m} \gamma_{m \uparrow}-\left(v_{R}^{m}\right)^{*} \gamma_{m \downarrow}^{\dagger}\right)\right]+ \text { h.c. }
\end{aligned}
$$


We treat two superconductors in the junction as a single system given by the state vector $|\operatorname{Gnd}(N)\rangle$, where $|\operatorname{Gnd}(N)\rangle$ satisfies

$$
\gamma_{n}|\operatorname{Gnd}(N)\rangle=0
$$

Note also that $|\operatorname{Gnd}(N)\rangle$ satisfies,

$$
e^{-\frac{i}{2} \hat{\chi}_{i}}|\operatorname{Gnd}(N)\rangle=e^{-\frac{i}{2} \chi_{i}}|\operatorname{Gnd}(N-1)\rangle
$$

Taking expectation value of $H_{L R}$ using $|\operatorname{Gnd}(N)\rangle$, we have the energy for the junction

$$
H_{J}^{e}=C \cos \left(\frac{e}{\hbar} \int_{R}^{L} d \mathbf{r} \cdot\left(\mathbf{A}^{\mathrm{em}}-\frac{\hbar}{2 e} \nabla \chi\right)+\alpha\right)
$$

where

$$
\frac{1}{2} C e^{i \alpha}=-2 T_{L R} \sum_{n} v_{L}^{n}\left(v_{R}^{n}\right)^{*}+\text { h.c. }
$$

Note that usually, the transfer of electron pairs is considered between the two superconductors in the Josephson junction using the second order perturbation theory with respect to $H_{L R}$; in this case, the supercurrent that flows without Bogoliubov excitations requires electron-pair tunneling, and the energy for the junction is given by

$$
H_{J}^{2 e}=C^{\prime} \cos \left(\frac{2 e}{\hbar} \int_{R}^{L} d \mathbf{r} \cdot\left(\mathbf{A}^{\mathrm{em}}-\frac{\hbar}{2 e} \nabla \chi\right)+\alpha^{\prime}\right)
$$

where $C^{\prime}$ and $\alpha^{\prime}$ are constants. This formula gives rise to the Ambegaokar-Baratoff relation [44] for the dc Josephson effect [43], and is valid for a weakly-coupled junction.

In the new formalism, $q=-e$ electron transfer is possible if the two superconductors in the junction is in a close contact and Equation (81) is valid; however, it is not possible in the standard formalism. Thus, from the view point of the re-derived result for the ac Josephson effect [21,24], the new formalism is more in accordance with the experiment.

In order to clarify the above point, re-investigations on the contact effect for the ac Josephson effect is necessary. In the new formalism, both $H_{J}^{e}$ and $H_{J}^{2 e}$ are possible, thus, there will be crossover of the junction properties whether it is described by $H_{J}^{e}$ or $H_{J}^{2 e}$ depending on the contact is weak or close. In this respect, it is noteworthy that un-paired electrons are more abundant than the standard theory in a Cooper pair box [45].

Lastly, we would like to point out a problem in the standard formalism from the view point of the decoherence by interaction with environment considered by Zurek [46]. The $U(1)$ gauge symmetry breaking origin of $\mathbf{A}^{\text {fic }}$ in the standard theory relies on the breaking of invariance for the global $U(1)$ phase change $c_{\mathbf{k} \sigma}^{\dagger} \rightarrow e^{i \alpha^{\prime \prime}} c_{\mathbf{k} \sigma}^{\dagger}\left(\alpha^{\prime \prime}\right.$ is a real constant) in $|\operatorname{BCS}(\mathbf{r} ; \chi)\rangle$. This breaking occurs because $|\operatorname{BCS}(\mathbf{r} ; \chi)\rangle$ is a linear combination of states with different particle numbers. However, such a state eventually becomes a mixed state due to the decoherence induced by the environment [46] since the relevant Hamiltonian conserves the particle number. In the resulting mixed state, the phase differences between the different particle number states are meaningless, thus, a physically meaningful phase $\chi$ or $\mathbf{A}^{\text {fic }}$ disappears sooner or later. Considering the fact that the supercurrent in a superconducting ring persist indefinitely, the physically meaningful $\mathbf{A}^{\text {fic }}$ should persist indefinitely; however, $|\operatorname{BCS}(\mathbf{r} ; \chi)\rangle$ does not have such $\mathbf{A}^{\text {fic }}$. On the other hand, $|\operatorname{Gnd}(\mathbf{r} ; N)\rangle$ with the stabilized $\mathbf{A}^{\text {fic }}$ has it.

Funding: This research received no external funding.

Conflicts of Interest: The author declares no conflict of interest. 


\section{References}

1. Dirac, P. Quantised singularities in the electromagnetic field. Proc. R. Soc. 1931, 133, 60.

2. Weinberg, S. Superconductivity for Particular Theorists Prog. Theor. Phys. Suppl. 1986, 86, 43. [CrossRef]

3. Bardeen, J.; Cooper, L.N.; Schrieffer, J.R. Theory of Superconductivity. Phys. Rev. 1957, 108, 1175. [CrossRef]

4. Bardeen, J. Theory of the Meissner Effect in Superconductors. Phys. Rev. 1955, 97, 1724. [CrossRef]

5. Anderson, P.W. Considerations on the Flow of Superfluid Helium Rev. Mod. Phys. 1966, 38, 298. [CrossRef]

6. Anderson, P.W. Basic Notions of Condensed Matter Physics;The Benjamin/Cummings Publishing Company, Inc.: San Francisco, CA, USA, 1984.

7. Bednorz, J.G.; Müller, K.A. Possible high Tc superconductivity in the Ba-La-Cu-O system. Z. Phys. B 1986, 64, 189. [CrossRef]

8. Anderson, P.W. The Theory of Superconductivity in the High-Tc Cuprates; Princeton Univ. Press: Princeton, NJ, USA, 1997.

9. Tranquada, J.M.; Woo, H.; Perring, T.G.; Goka, H.; Gu, G.D.; Xu, G.; Fujita, M.; Yamada, K. Quantum magnetic excitations from stripes in copper oxide superconductors. Nature 2004, 429, 534. [CrossRef]

10. Dean, M.P.M.; Dellea, G.; Springell, R.S.; Yakhou-Harris, F.; Kummer, K.; Brookes, N.B.; Liu, X.; Sun, Y.-J.; Strle, J.; Schmitt, T.; et al. Persistence of magnetic excitations in $\mathrm{La}_{2-x} \mathrm{Sr}_{x} \mathrm{CuO}_{4}$ from the undoped insulator to the heavily overdoped non-superconducting metal. Nat. Mater. 2013, 12, 1019. [CrossRef]

11. Sebastian, S.E. The Experiments by Y.-T.Hsu et al. Indicates the Existence of about 1nm Sized Vortices in a very Strong (45 T) Magnetic Field. Unpublished Result Reported at SNS2019, Tokyo, Japan, 2020.

12. Emery, V.J.; Kivelson, S.A. Importance of phase fluctuations in superconductors with small superfluid density. Nature 1995, 374, 434 [CrossRef]

13. Okazaki, A.; Wakaura, H.; Koizumi, H.; Ghantous, M.A.; Tachiki, M. Superconducting Transition Temperature of the Hole-Doped Cuprate as the Stabilization Temperature of Supercurrent Loops Generated by Spin-Twisting Itinerant Motion of Electrons. J. Supercond. Nov. Magn. 2015, 28, 3221. [CrossRef]

14. Morisaki, T.; Wakaura, H.; Koizumi, H. Effect of Rashba Spin-Orbit Interaction on the Stability of Spin-Vortex-Induced Loop Current in Hole-Doped Cuprate Superconductors: A Scenario for the Appearance of Magnetic Field Enhanced Charge Order and Fermi Surface Reconstruction. J. Phys. Soc. Jpn. 2017, 86, 104710. [CrossRef]

15. Bianconi, A.; Saini, N.L.; Lanzara, A.; Missori, M.; Rossetti, T.; Oyanagi, H.; Yamaguchi, H.; Oka, K.; Ito, T. Determination of the Local Lattice Distortions in the $\mathrm{CuO}_{2}$ Plane of $\mathrm{La}_{1.85} \mathrm{Sr}_{0.15} \mathrm{CuO}_{4}$. Phys. Rev. Lett. 1996, 76, 3412. [CrossRef] [PubMed]

16. Müller, K.A. Hand Book of High-Tempearture Superconductivity; Schrieffer, J.R., Brooks, J.S., Eds.; Springer: Berlin, Germany, 2007; p. 1.

17. Miyaki, S.; Makoshi, K.; Koizumi, H. Two-Copper-Atom Units Induce a Pseudo Jahn-Teller Polaron in Hole-Doped Cuprate Superconductors. J. Phys. Soc. Jpn. 2008, 77, 034702. [CrossRef]

18. Zhang, C.J.; Oyanagi, H. Local lattice instability and superconductivity in $\mathrm{La}_{1.85} \mathrm{Sr}_{0.15} \mathrm{Cu}_{1-x} \mathrm{M}_{x} \mathrm{O}_{4}\left(\mathrm{M}=\mathrm{Mn}_{\text {, }}\right.$ $\mathrm{Ni}$, and Co). Phys. Rev. B 2009, 79, 064521. [CrossRef]

19. Berry, M.V. Quantal phase factors accompanying adiabatic changes. Proc. R. Soc. Lond. Ser. A 1984, $391,45$.

20. Bohm, A.; Mostafazadeh, A.; Koizumi, H.; Niu, Q.; Zwanziger, J. The Geometric Phase in Quantum Systems; Springer: Berlin, Germany, 2003.

21. Koizumi, H. Spin-vortex Superconductivity. J. Supercond. Nov. Magn. 2011, 24, 1997. [CrossRef]

22. Koizumi, H.; Hidekata, R.; Okazaki, A.; Tachiki, M. Persistent current generation by the spin-vortex formation in cuprate with the single-valuedness constraint on the conduction electron wave functions. J. Supercond. Nov. Magn. 2014, 27, 121. [CrossRef]

23. Koizumi, H.; Okazaki, A.; Ghantous, M.A.; Tachiki, M. Supercurrent flow through the network of spin-vortetices in cuprates. J. Supercond. Nov. Magn. 2014, 27, 2435. [CrossRef]

24. Koizumi, H.; Tachiki, M. Supercurrent Generation by Spin-twisting Itinerant Motion of Electrons: Re-derivation of the ac Josephson Effect Including the Current Flow Through the Leads Connected to Josephson Junction. J. Supercond. Nov. Magn. 2015, 28, 61. [CrossRef]

25. Hidekata, R.; Koizumi, H. Spin-vortices and spin-vortex-induced loop currents in the pseudogap phase of cuprates. J. Supercond. Nov. Magn. 2011, 24, 2253. [CrossRef] 
26. Xia, J.; Schemm, E.; Deutscher, G.; Kivelson, S.A.; Bonn, D.A.; Hardy, W.H.; Liang, R.; Siemons, W.; Koster, G.; Fejer, M.M.; et al. Polar Kerr-Effect Measurements of the High-Temperature $\mathrm{YBa}_{2} \mathrm{Cu}_{3} \mathrm{O}_{6+x}$ Superconductor: Evidence for Broken Symmetry near the Pseudogap Temperature. Phys. Rev. Lett. 2008, 100, 127002. [CrossRef] [PubMed]

27. LeBoeuf, D.; Doiron-Leyraud, N.; Levallois, J.; Daou, R.; Bonnemaison, J.-B.; Hussey, N.E.; Balicas, L.; Ramshaw, R.J.; Liang, R.; Bonn, D.A.; et al. Electron pockets in the Fermi surface of hole-doped high-Tc superconductors. Nature 2007, 450, 533. [CrossRef] [PubMed]

28. Wang, Y.; Li, L.; Naughton, M.J.; Gu, G.D.; Uchida, S.; Ong, N.P. Field-Enhanced Diamagnetism in the Pseudogap State of the Cuprate $\mathrm{Bi}_{2} \mathrm{Sr}_{2} \mathrm{CaCu}_{2} \mathrm{O}_{8+\delta}$ Superconductor in an Intense Magnetic Field. Phys. Rev. Lett. 2005, 95, 247002. [CrossRef] [PubMed]

29. Damascelli, A.; Hussain, Z.; Shen, Z.-X. Angle-resolved photoemission studies of the cuprate superconductors. Rev. Mod. Phys. 2003, 75, 473. [CrossRef]

30. Koizumi, H. Explanation of Superfluidity Using the Berry Connection for Many-Body Wave Functions. J. Supercond. Nov. Magn. 2020. [CrossRef]

31. Bogoliubov, N.N. A New Method in the Theory of Superconductivity. I. Sov. Phys. JETP 1958, $34,41$. [CrossRef]

32. de Gennes, P.G. Superconductivity of Metals and Alloys; W. A. Benjamin, Inc.: New York, NY, USA, 1966.

33. Kane, C.L.; Mele, E.J. Z $Z_{2}$ Topological Order and the Quantum Spin Hall Effect. Phys. Rev. Lett. 2005, 95, 146802. [CrossRef] [PubMed]

34. Dirac, P.A.M. Section 70. In Principles of Quantum Mechanics, 4th ed.; Oxford Univ. Press: Oxford, UK, 1958.

35. Rashba, E.I. Properties of semiconductors with an extremum loop .1. Cyclotron and combinational resonance in a magnetic field perpendicular to the plane of the loop. Sov. Phys. Solid State 1960, 2, 1109.

36. Gutzwiller, M.C. Chaos in Classical and Quantum Mechanics; Springer: Berlin, Germany, 1990.

37. Sundaram, G.; Niu, Q. Wave-packet dynamics in slowly perturbed crystals: Gradient corrections and Berry-phase effects. Phys Rev. B 1999, 59, 14915. [CrossRef]

38. Dirac, P.A.M. Section 31. In Principles of Quantum Mechanics, 4th ed.; Oxford Univ. Press: Oxford, UK, 1958.

39. Schrödinger, E. Quantisierung als Eigenwertproblem. Ann. Phys. 1926, 79, 361. [CrossRef]

40. Kerman, A.K.; Koonin, S.E. Hamiltonian formulation of time-dependent variational principles for the many-body system. Ann. Phys. 1976, 100, 332. [CrossRef]

41. Onsager, L. Interpretation of the de Haas-van Alphen effect. Phil. Mag. Ser. 1952, 43, 1006. [CrossRef]

42. London, F. Superfluids; Wiley: New York, NY, USA, 1950; Voume 1.

43. Josephson, B.D. Possible new effects in superconductive tunnelling. Phys. Lett. 1962, 1, 251. [CrossRef]

44. Ambegaokar, V.; Baratoff, A. Tunneling Between Superconductors. Phys. Rev. Lett. 1963, 10, 486. [CrossRef]

45. Aumentado, J.; Keller, M.W.; Martinis, J.M.; Devoret, M.H. Nonequilibrium Quasiparticles and 2e Periodicity in Single-Cooper-Pair Transistors. Phys. Rev. Lett. 2004, 92, 066802. [CrossRef]

46. Zurek, W.H. Decoherence and the Transition from Quantum to Classical-Revisited. Los Alamos Sci. 2002, 27,86 .

(C) 2020 by the author. Licensee MDPI, Basel, Switzerland. This article is an open access article distributed under the terms and conditions of the Creative Commons Attribution (CC BY) license (http://creativecommons.org/licenses/by/4.0/). 